(2) Open Access Full Text Article

\title{
Anatomic and functional outcomes of retinectomy for the management of complicated retinal detachment with proliferative vitreoretinopathy
}

This article was published in the following Dove Press journal:

Therapeutics and Clinical Risk Management

3 October 2015

Number of times this article has been viewed

\author{
Paraschos Tranos' \\ Athanasios Vakalis' \\ Solon Asteriadis' \\ Evaggelos Lokovitis' \\ Ilias Georgalas ${ }^{2}$ \\ Panagiotis Stavrakas ${ }^{3}$ \\ 'Ophthalmica Eye Institute, \\ Thessaloniki, Greece; ${ }^{2}$ First \\ Department of Ophthalmology, \\ ${ }^{3}$ Second Department of \\ Ophthalmology, University of Athens \\ Medical School, Athens, Greece
}

\begin{abstract}
The aim of this study is to report the anatomic and functional outcomes of retinectomy for the management of rhegmatogenous retinal detachment complicated by proliferative vitreoretinopathy (PVR), comparing them with previously reported data and determining prognostic factors. Fifty-one eyes of 51 patients with established PVR grade $\mathrm{C}$ in which retinectomy was performed were retrospectively enrolled in the study. Primary outcome measures were anatomic success rate and final visual acuity. Secondary outcome measures were intraoperative complications, number of re-operations, and postoperative hypotony. Prognostic factors in relation to retinal re-attachment and final visual acuity were retrospectively analyzed. The rate of complete retinal re-attachment after one operation was $80 \%$ and after two operations it was $84 \%$. At the end of the follow-up, the macula was attached in $96 \%$ of the cases. Mean LogMAR best-corrected visual acuity (BCVA) improved significantly from $2.45 \pm 0.66$ preoperatively to $1.37 \pm 0.75$ at the end of the follow-up $(P<0.001)$. BCVA improved in 37 eyes $(72 \%)$, remained the same in eleven eyes $(22 \%)$ and worsened in three eyes $(6 \%)$. Postoperative hypotony was observed in $2 \%$ of cases. Postoperative BCVA was significantly correlated with preoperative BCVA $(P<0.001)$, extension of PVR $(P=0.013)$, preoperative use of gas instead of silicone oil (SO) $(P=0.01)$, and removal of SO $(P=0.05)$. SO was left in situ in $35 \%$ of eyes. In conclusion, retinectomy is a surgical option providing good anatomical and reasonable visual outcomes in complicated retinal detachment with PVR. Better preoperative visual acuity, lesser extension of PVR, and the use of gas tamponade during the primary repair are relevant factors for a successful outcome whereas re-proliferation of epiretinal membranes seems to be the main reason of anatomical failure.
\end{abstract}

Keywords: retinal detachment, proliferative vitreoretinopathy, vitrectomy, retinectomy

\section{Introduction}

Proliferative vitreoretinopathy (PVR) is a serious complication of rhegmatogenous retinal detachment (RRD) that often requires a complex surgical management. Several techniques have been described to treat retinal detachment (RD) associated with proliferative membranes, with or without coexisting anterior shortening of the retinal surface. These techniques may involve extensive membrane dissection and peeling, combined vitrectomy with buckle procedures, the performance of inferior circumferential retinectomy/retinotomy, radial retinotomy, or 360 degrees retinectomy in severe cases. Most of studies evaluating these techniques have shown the achievement of satisfactory attachment rates postoperatively. ${ }^{1-7}$ Retinotomy and retinectomy were first introduced by Machemer in 1979 and were reserved for the treatment of selected RDs complicated with severe PVR when other methods had failed to achieve retinal
Correspondence: Panagiotis Stavrakas Second Department of Ophthalmology, University of Athens Medical School, I Rimini Street, Chaidari, I 2462 Athens, Greece

Tel +3069442766I0

Email panos.stavrakas@yahoo.com 
re-attachment. ${ }^{8}$ In recent years, there is a general trend toward the use of vitrectomy as a first option for the management of RRDs and retinectomy has gained consideration as a procedure of choice in the setting of anterior retinal shortening and grade C PVR. ${ }^{9}$ Previous studies reporting in retinectomy results have included complicated RDs originating from ocular trauma, tractional RDs, acute retinal necrosis and endophthalmitis, cases of notably higher risk of surgical failure. ${ }^{6,7}$ The purpose of this study is to evaluate and report the anatomical and functional outcomes of eyes undergoing retinectomy for the management of purely primary RRD with grade C PVR, ${ }^{10}$ to determine prognostic factors and to compare results with existing data in the literature.

\section{Materials and methods}

Fifty-one eyes of 51 consecutive patients undergoing surgery for RRD at a university teaching center and a private clinical center specialized in retinal disorders were included in the study. All eyes had anterior PVR grade $\mathrm{C}$ according to the classification developed by the Retina Society. ${ }^{10}$ Additionally, some of these cases had posterior PVR grade C. Permission to conduct the study was obtained from the Research and Ethics committees of our Institutions. The case notes of the consecutive patients who underwent surgery between January 2009 and February 2013 were retrospectively retrieved and analyzed. Patients were fully informed of all aspects of the study and all provided written informed consent in accordance to the principles of Helsinki and good clinical practice guidelines. The data analysis included the evaluation of baseline characteristics, preoperative, intraoperative, and postoperative parameters. Baseline and preoperative characteristics included age, sex, laterality, ocular co-morbidities, duration of symptoms, number of previous operations, type and duration of tamponade, crystalline lens status, and best-corrected visual acuity (BCVA). Intraoperative and postoperative parameters included the extension (clock hours) of retinectomy, type of retinopexy, time of removal of the endotamponade, further reoperations, overall number of retinectomies, intraocular pressure (IOP), complications, and final BCVA. Main outcome measures were retinal re-attachment rate and final BCVA and secondary outcome measures included intraoperative complications, number of re-operations, and IOP changes. Exclusion criteria for the study were an age under 18 years and previous retinectomies. The study was performed in accordance with the tenets of the Declaration of Helsinki.

All operations were performed using a standard $20 \mathrm{G}$ (34 eyes) and 23G (17 eyes) three-port pars plana vitrectomy system. All eyes underwent vitrectomy with trimming of the vitreous up to the vitreous base. Meticulous removal of all preretinal membranes was performed with forceps after staining them with membrane blue (DORC, Zuidland, the Netherlands). Delamination and segmentation of PVR membranes were performed starting from the posterior membranes and extending up anteriorly to the equator. After the removal of the membranes, intraocular diathermy was applied to the retina at the edge of the retinectomy area. Retinectomy was performed using vertical scissors or a vitreous cutter. In some cases, IOP elevation was required to obtain hemostasis and minimize bleeding from the vessels at the edge of the retinectomy. Retinectomy was always circumferential and extended as many clock hours as necessary to relieve all tractions and the anterior retinal shortening. In most cases, an extension of the retinectomy of at least 6 clock hours (from 3 to 9 clock hours) was intended based on the assumption that such extension would contribute significantly to the relieve of the circumferential traction. Upon completion of the retinectomy, a bubble of perfluoro-n-octane (PFCL) was injected into the posterior pole up to the retinectomy edge and peripheral non-functioning retina was removed with vitreous cutter to prevent neovascularization and re-proliferation. Additional PFCL was then injected into the eye up to the ora serrata and laser retinopexy was applied to the posterior edge of the retinectomy. Laser spots were placed in a confluent fashion in three to five rows. In some cases, additional cryopexy was applied at the lateral edges of the retinotomy based on the preference of surgeon. Finally, PFCL was directly exchanged for silicone oil (SO) 5,700 or 1,300 cS. In aphakic eyes, a peripheral iridectomy was done inferiorly with a vitreous cutter.

Postoperatively, patients were instructed to posture with their face down for 1 day. In the following 10 days, patients were asked to avoid gazing up during daytime and to sleep on either cheek at night. Removal of SO was performed at least 3 months after retinectomy. SO was not removed from eyes that had hypotony, which was defined as an IOP of less than $5 \mathrm{mmHg}$. Likewise, SO was not removed from eyes with low IOP, defined as an IOP value between 6 and $10 \mathrm{mmHg}$ because these eyes were considered to be at high risk of developing severe hypotony.

Statistical analysis was performed with SPSS software for Windows (version 16.0, SPSS, Inc, Chicago, IL, USA). Snellen visual acuity was converted to LogMAR scale for the statistical analysis. Relations between categorical variables were evaluated using the $\chi^{2}$ test. The distribution of all variables was examined using frequency tables and descriptive statistics. Box-and-whisker plots and histograms were used to display the different data distributions. Parametric methods including independent Student's $t$-test, ANOVA, and linear regression were used for normally distributed variables. Non-parametric 
tests such as Mann-Whitney $U$ and Wilcoxon signed rank tests were used for variables that did not show normality. The relationship of the final BCVA with a great variety of prognostic factors was examined using the Spearman rank correlation test. The relationship between categorical variables was evaluated using the chi-square test. In addition, a multivariate model was applied to evaluate the relationship between functional and anatomical results with several explanatory variables simultaneously. All tests of association were considered to be statistically significant if $P<0.05$.

\section{Results}

A total of 51 consecutive eyes with established PVR grade $\mathrm{C}$ secondary to RRD were included in the study. Thirty-two patients were males and 19 were females with a median age of 61 years (range, 18-87 years). The cohort included a similar number of right (47\%) and left eyes (53\%). Seventeen eyes were phakic, 33 were pseudophakic, and one eye was aphakic. All cases had a minimum follow-up of 12 months (range, 12-48 months). Table 1 summarizes the preoperative characteristics of the eyes included in the study.

Mean preoperative BCVA was 2.45 \pm 0.66 LogMAR. Eight eyes (15.6\%) had a macula-on RRD and in 43 eyes $(84.3 \%)$ the macula was already detached at the time of

Table I Preoperative characteristics

\begin{tabular}{|c|c|}
\hline \multicolumn{2}{|l|}{ Baseline characteristics $(\mathbf{N}=5 \mathrm{I})$} \\
\hline Age, median, years (range) & 61 (18-87) \\
\hline \multicolumn{2}{|l|}{ Sex } \\
\hline Male & 32 \\
\hline Female & 19 \\
\hline \multicolumn{2}{|l|}{ Etiology } \\
\hline RRD & 51 \\
\hline \multicolumn{2}{|l|}{ Lens status } \\
\hline Phakic & 17 \\
\hline Pseudophakic, aphakic & 33,1 \\
\hline \multicolumn{2}{|l|}{ Macula status at time of retinectomy } \\
\hline \multicolumn{2}{|l|}{ Number (\%) } \\
\hline Macula on & $8(15.6 \%)$ \\
\hline Macula off & $43(84.3 \%)$ \\
\hline Preop BCVA, LogMAR & $2.45( \pm 0.66)$ \\
\hline No of previous RRD operations, mean (range) & I.II (I-3) \\
\hline Previous vitrectomy & 51 \\
\hline Buckle & 0 \\
\hline \multicolumn{2}{|l|}{ Previous endotamponade used } \\
\hline Gas & $16(31.4 \%)$ \\
\hline Silicone oil & $35(68.6 \%)$ \\
\hline Extend of RRD, clock hours, mean (range) & $10(6-12)$ \\
\hline Extend of PVR, clock hours & $7.32( \pm 3.6)$ \\
\hline Anterior PVR & $51(100 \%)$ \\
\hline Anterior and posterior & $14(27.5 \%)$ \\
\hline Follow-up, months, minimum (range) & $12(12-48)$ \\
\hline
\end{tabular}

Therapeutics and Clinical Risk Management 20 I5:I I surgery. All eyes had at least one failed surgical procedure not achieving a retinal re-attachment prior to the retinectomy procedure (mean: 1.11, range: 1-3). As intraocular tamponade prior to retinectomy, $\mathrm{SO}$ was used in 35 eyes $(68.6 \%)$ and gas in 16 eyes (31.4\%). Mean extension of RD was 10 clock hours (range, 6-12 clock hours) and mean extension of PVR was 7.32 \pm 3.6 clock hours. Anterior PVR was present in all cases, with additional coexisting posterior proliferation in 14 eyes (27.5\%). Preretinal membrane peeling (PVR peeling) prior to retinectomy was attempted in all eyes, but in eleven patients complete dissection was not possible due to the immature nature of membranes.

Table 2 summarizes the postoperative outcomes of the study. Laser photocoagulation was only used for retinopexy in 20 eyes whereas a combined laser and cryopexy treatment was applied to the lateral edges of retinectomy in 31 eyes. Re-attachment of the retina after one surgical procedure was observed in $41 / 51(80 \%)$ eyes. Non-progressive inferior detachment under SO was observed in six eyes (12\%). In these six cases, no further surgeries were performed as the extension and stability of the RD did not influence the visual acuity. Retinal re-detachment involving the macula occurred in four eyes $(8 \%)$ because of re-occurrence of PVR. These eyes were managed by performing an additional retinectomy, membrane dissection, and re-insertion of SO. Final retinal re-attachment was achieved in two cases whereas the other two patients declined additional procedures (Figure 1).

SO removal was performed in 33 eyes $(65 \%)$ and left in situ in 18 eyes (35\%) after one or more surgical procedures aimed at achieving retinal re-attachment. The decision for

Table 2 Final outcomes

\begin{tabular}{|c|c|}
\hline \multicolumn{2}{|l|}{ Final results (postoperative, final FU) } \\
\hline Primary complete retinal attachment & $4 \mathrm{I} / 5 \mathrm{I}(80 \%)$ \\
\hline Final complete retinal attachment & $43 / 5 I(84 \%)$ \\
\hline Final macular attachment & $49 / 5 I(96 \%)$ \\
\hline Silicone oil removal & $33 / 5 I(65 \%)$ \\
\hline With chronic oil & $|8 / 5|(35 \%)$ \\
\hline Final visual acuity, median (LogMAR) & $1.37 \pm 0.75(1.3)$ \\
\hline Visual acuity with retained SO & $1.52 \pm 0.7$ \\
\hline Visual acuity after removal of SO & $0.9 \pm 0.7$ \\
\hline Non-progressive inferior RD (not requiring surgery) & $6 / 51(12 \%)$ \\
\hline Retinal macular re-detachment & $4 / 51(8 \%)$ \\
\hline \multicolumn{2}{|l|}{ Retinectomy number } \\
\hline I & 47 \\
\hline$>1$ & 4 \\
\hline \multicolumn{2}{|l|}{ Postoperative IOP } \\
\hline $0-5 \mathrm{mmHg}$ & $|/ 5|(2 \%)$ \\
\hline $6-10 \mathrm{mmHg}$ & $9 / 51(18 \%)$ \\
\hline$|\mathrm{I}-2| \mathrm{mmHg}$ & $4|/ 5|(8 I \%)$ \\
\hline Postoperative glaucoma & $2 / 51(3.9 \%)$ \\
\hline
\end{tabular}




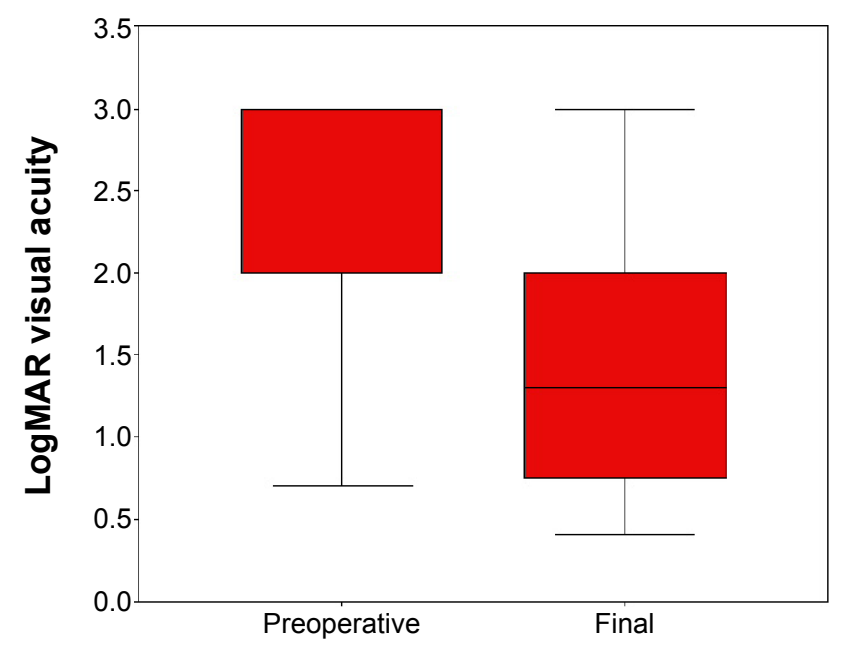

Figure I Box plot chart of preoperative and final postoperative LogMAR BCVA. Abbreviation: BCVA, best-corrected visual acuity.

leaving SO in situ was based upon patients' preference who declined to undertake the risk of retinal re-detachment (8/18 eyes), whereas in ten cases low IOP $(\leq 10 \mathrm{mmHg})$ prohibited further surgery for removal of SO.

Mean preoperative BCVA improved significantly $(P<0.001)$ from $2.45 \pm 0.66$ (median 2.27$)$ to $1.37 \pm 0.75$ (median 1.3) LogMAR at the last postoperative visit (Figure 2). BCVA improved in 37 eyes (72\%), remained the same in $11(22 \%)$ eyes, and became worse in three eyes $(6 \%)$. Although the mean final LogMAR BCVA was significantly better in those eyes that underwent an additional surgery to remove the SO compared with eyes in which the SO was retained $(0.90 \pm 0.70$ vs $1.52 \pm 0.70, P=0.003)$, the change in LogMAR visual acuity was similar in both groups $(0.92 \pm 0.66$ vs $1.02 \pm 0.72, P=0.65)$.

Hypotony, defined as an IOP of less than $5 \mathrm{mmHg}$, was present in one eye (2\%). Nine eyes $(18 \%)$ had an IOP between 6 and $10 \mathrm{mmHg}$ and 41 eyes $(81 \%)$ had a pressure

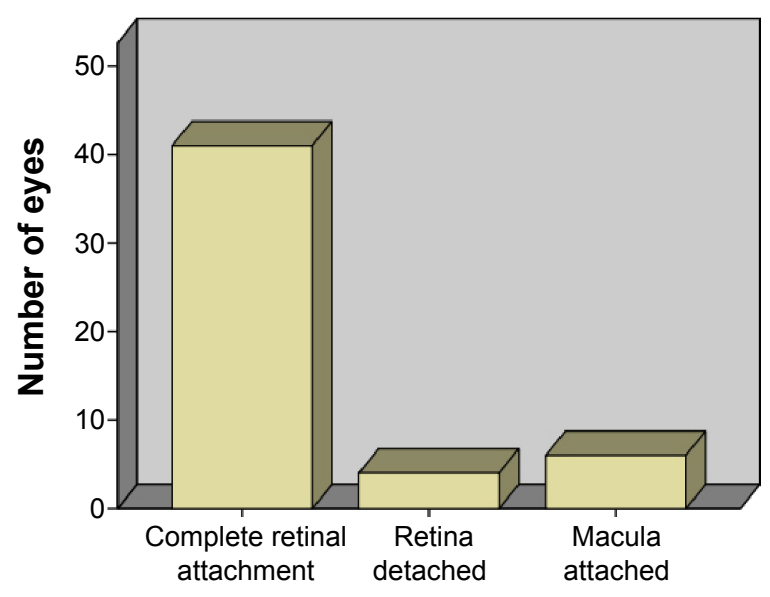

Figure 2 Retinal anatomic status following the first retinal re-attachment surgery with retinectomy.
Table 3 Preoperative and intraoperative factors significantly associated with better final best-corrected visual acuity

Correlation between final BCVA and pre and intraoperative parameters*

\begin{tabular}{ll}
\hline Preoperative BCVA & $P<0.001$ \\
Extension of PVR & $\begin{array}{l}P=0.013 \\
\text { Use of gas as endotamponade during primary retinal }\end{array}$ \\
$\begin{array}{l}P=0.01 \\
\text { detachment repair } \\
\text { Removal of silicone oil }\end{array}$ & $P=0.003$ \\
\hline $\begin{array}{l}\text { Notes: *Spearman rank correlation test. Statistical significance for } P<0.05 . \\
\text { Abbreviations: BCVA, best-corrected visual acuity; PVR, }\end{array}$ \\
vitreoretinopathy.
\end{tabular}

between 10 and $21 \mathrm{mmHg}$. Only two eyes required long-term IOP-lowering medication without the need of further filtration surgery. The eye with hypotony did not show any related clinical signs in terms of macular folds or corneal Descemet fold, and the retina was fully attached. No statistically significant correlations were found between IOP and the extension of retinectomy or the extension of PVR $(P>0.05)$.

At the end of the follow-up, final BCVA was found to have a positive and statistically significant correlation with preoperative BCVA $(P<0.001)$, extension of PVR $(P=0.013)$, preoperative use of gas instead of SO $(P=0.01)$, and removal of SO $(P=0.003)$ (Table 3$)$. Additional factors were also tested, such as the extension of retinectomy, the number of surgeries, and the duration of SO tamponade, but no statistically significant correlations were found between the aforementioned factors and the final BCVA.

Regarding postoperative complications, there were four cases with inadvertent iatrogenic breaks occurring during the PVR peeling, and six eyes with retained PFCL after surgery. In five of these six eyes, PFCL was found at the subretinal level, involving the posterior pole in three cases and detected outside the temporal arcades in two eyes. The retained PFCL did not affect visual acuity in any of cases and for this reason no additional surgical procedures were attempted for its removal. Other possible intraoperative complications including vitreous or suprachoroidal hemorrhage or iatrogenic posterior pole trauma were not observed.

\section{Discussion}

Cellular proliferation and contractile preretinal membrane formation are the main causes of failure in RD surgery. ${ }^{11}$ The frequency of PVR has been reported to be of $5 \%-12 \%$ in cases of $\mathrm{RD} .{ }^{12}$ The complete removal of preretinal membranes in such cases is not always possible, especially when anterior PVR is present, and alternative techniques such as retinectomy may be necessary to obtain a good anatomical and visual outcome. In our series, the primary anatomic success rate was $80 \%$, with a final complete re-attachment rate of $84 \%$. Macula 
attachment was present in 49 eyes (96\%) at the end of the follow-up. All these rates were higher or consistent with those from previous studies evaluating the outcomes of retinectomy, reporting success rates between $47 \%$ and $95 \%$. $^{1,2,4,6,7,9,13-15}$ Nevertheless, comparison between different studies on retinectomy is problematic because of significant differences in the inclusion criteria and surgical techniques. Specifically, we have only included primary RRDs whereas other studies have included cases with perforating trauma or tractional RDs with notably higher risk for re-detachment. ${ }^{2,4,6,7,13,14,16-18}$ In addition, the extension of retinectomy was 180 degrees in most of our cases in contrast to previously reported series that extended the retinectomy to 360 degrees by default. ${ }^{1,16}$ Moreover, no comparable results regarding the timing of retinectomy have been reported in the peer-reviewed literature and this factor has been shown to play an important role on the final success rate. ${ }^{9}$ In the current study, the surgical approach was more proactive, performing a primary retinectomy in the presence of any early anterior shortening. This factor combined with our inclusion criteria and our lower rate of SO removal may have contributed to the high success rate of macular attachment found in our study.

Mean BCVA improved significantly after surgery, with more than $70 \%$ of eyes experiencing a visual improvement following surgery and $45 \%$ of them achieving a BCVA of $6 / 60$ or better. In previous studies, functional success ranged from $25 \%$ to $68 \%$ when it was defined as a postoperative BCVA of $5 / 200$ or better, ${ }^{6,9,18}$ and between $10 \%$ and $51 \%$ when it was defined as a postoperative BCVA of 20/200 or better. ${ }^{1,2,6,7,13,18}$ Grigoropoulos et al reported a visual stabilization or improvement in approximately $70 \%$ of cases in a series of 304 eyes, and Tan et al reported a success rate of $60 \%$ for primary retinectomy. ${ }^{6,9}$ In this study, re-proliferation of preretinal membranes was the main reason of failure of the retinectomy procedure. Re-occurrence of PVR was observed mainly near the site of retinectomy causing further retinal shortening or adjacent to areas of incomplete membrane dissection during the primary procedure.

The relatively high anatomical and functional success rate presented herein fully justifies the selection of an aggressive surgical technique such as retinectomy in eyes with RRD complicated by PVR. This is also supported by Brown et al who found a statistically significant gain of quality-adjusted life-years per treated patient in eyes undergoing vitreoretinal surgery for the management of RD complicated by severe PVR compared with no treatment. ${ }^{19}$ This procedure for the management of PVR associated with RD is cost-effective when compared with other widely accepted interventional therapies across diverse medical specialties. ${ }^{19}$
In the current study, we have also tried to identify prognostic factors for the functional and anatomical outcome of the surgical procedure evaluated. Previous studies have reported a negative association between the extension of retinectomy and the visual outcome. Such relation was expected considering that the size of retinectomy was related to the severity of posterior PVR. ${ }^{6,9,13}$ Our data showed that final visual outcome was positively related to the preoperative visual acuity, the removal of SO, and the use of gas instead of SO as endotamponade in previous surgeries. Additionally, we found that final visual acuity was negatively related to the preoperative extension of PVR. All these factors correlated to the final visual outcome seem to represent the severity of PVR, as eyes with better preoperative BCVA are more likely to have less advanced PVR and shorter duration of macular detachment, to require less demanding surgical procedures and to be suitable for earlier removal of SO. The use of gas in the primary surgical procedure usually implies a less complicated $\mathrm{RD}$, which is usually associated with a more favorable prognosis. As in most of previous studies, no correlations were found between the extension of retinectomy and the anatomic success, although the number of eyes in our series may provide a lower statistical power than in previous studies, leading to the lack of statistical significance in the analysis of correlations. ${ }^{6,9,13,14,20}$

In this study, re-proliferation of preretinal membranes was the main reason of failure of the retinectomy procedure. Re-occurrence of PVR was observed mainly near the site of retinectomy causing further retinal shortening or adjacent to areas of incomplete membrane dissection during the primary procedure.

Hypotony is a well-described complication of retinectomy surgery. It may complicate eyes with SO endotamponade or increase the severity of the disease after removal of SO. The exact mechanism of hypotony is not easily identified and there may be one or more contributing factors. The exposure of bare retinal pigment epithelium and choroid may increase the amount of uveoscleral outflow in the presence of normal aqueous production. ${ }^{21}$ For this reason, retinectomy has been proposed as an effective treatment for intractable glaucoma. ${ }^{21}$ Based on this assumption, there could be a relation between the extension of retinectomy and the degree of hypotony, as supported by Kirchhof. ${ }^{22}$ In our series, this relationship was not observed. Hypotony may also be attributable to anterior PVR and contraction of cyclitic membranes leading to atrophy of the ciliary body. In order to minimize the risk for such complication, a meticulous removal of the anterior to the retinotomy retinal flap in conjunction with the removal of cyclitic membranes was performed in our series, and therefore all residual tractions to the ciliary body were eliminated. 
This may explain the low percentage of hypotony observed in our series $(2 \%)$.

Our data did not elicit any correlation between the grade or extension of PVR and postoperative hypotony. The comparison of our data with outcomes previously reported in the literature is not an easy task as the definition of hypotony varies significantly among authors. Previously reported hypotony rates in retinectomy studies vary from $4.1 \%$ to $43 \% .^{6,9,13,20}$ Nevertheless, in our study, a relatively low IOP defined as an IOP value between 6 and $10 \mathrm{mmHg}$ was found in nine patients $(17.6 \%)$. In such cases, the removal of the SO was not possible because of the high risk of postoperative hypotony, which complicates the management of these cases and limits potential further visual improvements.

In $46 \%$ of our cases, cryopexy was performed at the edges of the retinectomy area as well as laser photocoagulation. Cryopexy is widely suspected to produce a higher degree of inflammation compared with laser retinopexy and be a predisposing factor for the development of postoperative PVR in eyes at high risk. ${ }^{23,24}$ In our series, we did not observe any relationship between the use of cryopexy and the development of macular pucker under SO. Likewise, a higher redetachment rate was not found in the cryopexy group.

This study has some limitations that should be mentioned. The retrospective collection of the data is the most important limitation. In addition, no direct comparison with a control group including eyes undergoing combined vitrectomy and scleral buckle surgery for the treatment of advanced PVR was performed. Furthermore, future studies with larger sample sizes should be conducted to confirm the results of this series.

In conclusion, the results presented herein support that retinectomy as a primary procedure is associated with a favorable anatomical outcome and acceptable visual recovery considering the severity of the disease. Based on our results, we postulate that early intervention may have an important role on obtaining high anatomical and functional success rates. Future studies comparing primary retinectomy with combined procedures in PVR cases in terms of clinical outcomes and patient recovery should be performed.

\section{Disclosure}

The authors report no conflicts of interest in this work.

\section{References}

1. Quiram PA, Gonzales CR, Hu W, et al. Outcomes of vitrectomy with inferior retinectomy in patients with recurrent rhegmatogenous retinal detachments and proliferative vitreoretinopathy. Ophthalmology. 2006; 113(11):2041-2047.
2. Faude F, Lambert A, Wiedemann P. 360 degrees retinectomy in severe anterior PVR and PDR. Int Ophthalmol. 2004;22(2):119-123.

3. Morel C, Doan S, Rivoal O, Ameline B, Monin C, Larricart P. Relaxing retinopathies and liquid perfluorocarbons. $J$ Fr Ophthalmol. 1998;21(5): 315-320.

4. Metge F, Massin P, Gaudric A. Retinectomies in the treatment of retinal detachments with vitreoretinal proliferation. J Fr Ophthalmol. 1997; 20(5):345-349.

5. Lu L, Gao R, Lin X. The application of retinotomy and retinectomy for complicated retinal detachment. Yan Ke Xиe Bao. 1998;14(2):94-96.

6. Grigoropoulos VG, Benson S, Bunce C, Charteris DG. Functional outcome and prognostic factors in 304 eyes managed by retinectomy. Graefes Arch Clin Exp Ophthalmol. 2007;245(5):641-649.

7. Lim AK, Alexander SM, Lim KS. Combined large radial retinotomy and circumferential retinectomy in the management of advanced proliferative vitreoretinopathy. Retina. 2009;29(1):112-116.

8. Machemer R. Retinotomy. Am J Ophthalmol. 1981;92:768-774.

9. Tan HS, Mura M, Lesnik Oberstein SY, De Smet MD. Primary retinectomy in proliferative vitreoretinopathy. Am J Ophthalmol.2010;149(3): 447-452.

10. Machemer R, Aaberg TM, Freeman HM, Irvine AR, Lean JS, Michels RM. An updated classification of retinal detachment with proliferative vitreoretinopathy. Am J Ophthalmol. 1991;112(2):159-165.

11. Charteris DG, Downie J, Aylward GW, Sethi C, Luthert P. Intraretinal and periretinal pathology in anterior proliferative vitreoretinopathy. Graefes Arch Clin Exp Ophthalmol. 2007;245(1):93-100.

12. Charteris DG, Sethi CS, Lewis GP, Fisher SK. Proliferative vitreoretinopathy-developments in adjunctive treatment and retinal pathology. Eye. 2002;16(4):369-374.

13. Han DP, Lewis MT, Kuhn EM, et al. Relaxing retinotomies and retinectomies. Surgical results and predictors of visual outcome. Arch Ophthalmol. 1990;108(5):694-697.

14. Bovey EH, De Ancos E, Gonvers M. Retinotomies of 180 degrees or more. Int Ophthalmol. 1998-1999;22(2):119-123.

15. Machemer R, McCuen BW 2nd, de Juan E Jr. Relaxing retinotomies and retinectomies. Am J Ophthalmol. 1986;102(1):7-12.

16. Kolomeyer AM, Grigorian RA, Mostafavi D, Bhagat N, Zarbin MA. 360 retinectomy for the treatment of complex retinal detachment. Retina. 2011;31(2):266-274.

17. Banaee T, Hosseini SM, Eslampoor A, Abrishami M, Moosavi M. Peripheral 360 retinectomy in complex retinal detachment. Retina. 2009;29(6):811-818.

18. Tseng JJ, Barile GR, Schiff WM, Akar Y, Vidne-Hay O, Chang S. Influence of relaxing retinotomy on surgical outcomes in proliferative vitreoretinopathy. Am J Ophthalmol. 2005;140(4):628-636.

19. Brown GC, Brown MM, Sharma S, Busbee B, Landy J. A cost-utility analysis of interventions for severe proliferative vitreoretinopathy. Am J Ophthalmol. 2002;133(3):365-372.

20. Morse LS, McCuen BW 2nd, Machemer R. Relaxing retinotomies. Analysis of anatomic and visual results. Ophthalmology. 1990;97(5): 642-647.

21. Joussen AM, Walter P, Jonescu-Cuypers CP, et al. Retinectomy for treatment of intractable glaucoma: long term results. Br J Ophthalmol. 2003;87(9):1094-1102.

22. Kirchhof B. Retinectomy lowers intraocular pressure in otherwise intractable glaucoma: preliminary results. Ophthalmic Surg. 1994;25(4): 262-267.

23. Nagasaki H, Shinagawa K, Mochizuki M. Risk factors for proliferative vitreoretinopathy. Prog Retin Eye Res. 1998;17(1):77-98.

24. Bonnet M, Fleury J, Guenoun S, Yaniali A, Dumas C, Hajjar C. Cryopexy in primary rhegmatogenous retinal detachment: a risk factor for postoperative proliferative vitreoretinopathy? Graefes Arch Clin Exp Ophthalmol. 1996;234(12):739-743. 
Therapeutics and Clinical Risk Management

Dovepress

\section{Publish your work in this journal}

Therapeutics and Clinical Risk Management is an international, peerreviewed journal of clinical therapeutics and risk management, focusing on concise rapid reporting of clinical studies in all therapeutic areas, outcomes, safety, and programs for the effective, safe, and sustained use of medicines. This journal is indexed on PubMed Central, CAS,

Ease, Scopus and the Elsevier Bibliographic databases. The manuscript management system is completely online and includes a very quick and fair peer-review system, which is all easy to use. Visit http://www.dovepress.com/testimonials.php to read real quotes from published authors.

Submit your manuscript here: http://www.dovepress.com/therapeutics-and-clinical-risk-management-journal 